\title{
El e-portfolio como herramienta para el desarrollo profesional docente
}

\author{
Alicia Martínez-De la Muela ${ }^{a}$, Mercedes García-Garcíab y Blanca Arteaga-Martínez \\ aUniversidad Internacional de La Rioja (UNIR), alicia.martinez@unir.net, bUniversidad Complutense \\ de Madrid, mergarci@edu.ucm.es y 'Universidad Internacional de La Rioja (UNIR) \\ blanca.arteaga@unir.net
}

\begin{abstract}
This study considers the usefulness of the digital portfolio or e-portfolio as a tool for professional educational development. The purpose is to contribute to teachers training processes with an instrument of evaluation, promoting a culture of reflection. The investigation is based on an online workshop, directed to 150 teachers and headmasters from schools that are in a moment of change and transformation of their methodologies and learning culture. The formation points out critical thinking, reflection and teamwork as the key competences in a school of the 21st century. At the same time, information and communication technologies have been touted as potentially powerful tools for educational change and reform; making learning a visible, engaging and active process connected to their teaching career. For the withdrawal of information an ad hoc questionnaire is used, as well as the utilization of the own instrument where the improved competitions are analyzed. The outcomes reveal how teachers and headmasters perceive the use of the portfolio for professional educational development, reflection and advantages and limitations of the production of the tool for his professional development.
\end{abstract}

Keywords: Formation, professional educational development, competitions, evaluation, portfolio online, innovation

\footnotetext{
Resumen

El estudio plantea la utilidad del portfolio digital o e-portfolio como herramienta para el desarrollo profesional docente. El objetivo es aportar un instrumento de evaluación para la formación docente, que favorezca una cultura de reflexión. Para ello se desarrolla un curso online dirigido a 150 docentes y directivos de centros educativos, inmersos en un momento de
} 
El e-portfolio como herramienta para el desarrollo profesional docente

cambio y de transformación en sus metodologías de aprendizaje. La formación se centra en la reflexión sobre la práctica docente, que junto al razonamiento crítico y el trabajo en equipo se consolidan como competencias principales del centro educativo. Los recursos digitales facilitan la elaboración del portfolio frente a otros medios tradicionales, aportando las evidencias de aprendizaje en diferentes formatos y ayudando a la retroalimentación formativa. Para la recogida de datos se utiliza un cuestionario ad hoc, así como la utilización del e-portfolio donde se analizan las competencias mejoradas. Los resultados recogen la percepción de docentes y directores respecto al uso del portfolio para su desarrollo profesional docente, centrando los datos en la capacidad reflexiva y las ventajas y limitaciones de la elaboración de la herramienta para su desarrollo profesional.

Palabras clave: formación, desarrollo profesional docente, competencias, evaluación, e-portfolio, innovación

\section{Introducción}

El desarrollo profesional docente se entiende como un proceso donde los profesionales de la educación "revisan, renuevan y desarrollan su compromiso como agentes de cambio, con los propósitos morales de la enseñanza y mediante los cuales adquieren y desarrollan conocimientos, habilidades e inteligencia emocional” (Day, 1999: 4). El desarrollo profesional docente es considerado una herramienta imprescindible para la mejora escolar y profesional, comprometida con el cambio y la innovación (Marcelo, 2011). Este proceso de formación continua no debe quedarse en unas meras actividades formativas, sino que precisa de un sistema de evaluación que permita a los docentes convertirse en mejores profesionales.

Se entiende por competencias "el conjunto de conocimientos, capacidades, habilidades y actitudes necesarias para realizar actividades diversas con un cierto nivel de calidad y eficacia” (Bisquerra, 2003: 14). El desarrollo profesional docente implica la formación en competencias, relacionándolas con la persona y con lo que ésta es capaz de lograr (Tunning, 2003), que permitan a los docentes llevar a cabo su práctica educativa de forma más eficaz y adaptada a la realidad. Según Imbernón (1994:11) "unir la formación al desarrollo profesional es ver la formación como un aprendizaje constante, acercando ésta al desarrollo de actividades profesionales y a la práctica profesional”. La evaluación del desarrollo profesional docente se debe poner en el centro de la investigación, dirigida a unos profesionales que se capaciten mejor en competencias. En este trabajo se han seleccionado algunas de las competencias consideradas como genéricas; trabajo en equipo 
(competencia interpersonal) y práctica reflexiva y crítica (competencia instrumental) como necesarias para un adecuado desarrollo profesional de los docentes, permitiéndoles desarrollar su práctica educativa en el aula con más seguridad y dando respuesta a los cambios del sistema educativo.

El aprendizaje a lo largo de la vida (LLL), competencia central defendida en el Espacio Europeo de Educación Superior (EEES), hace que la persona sea el centro del aprendizaje, actuando con autonomía. La excesiva información hace que sea necesario el desarrollo de habilidades que permitan adecuarse a las situaciones cambiantes (García, 2008). La importancia de la práctica, la colaboración, la posición activa y reflexiva del profesorado en el proceso de desarrollo profesional es valorada por diversos autores (González Maura, 2006; Peña, 2003).

En el EEES, los docentes han de convertir su práctica evaluativa en una oportunidad para desarrollar aprendizajes útiles. Éste es un aspecto clave de la teoría de evaluación orientada al aprendizaje al defender que: los estudiantes tienen que participar en la evaluación, las tareas de evaluación tienen que ser auténticas, realistas, y proactivas, y se debe favorecer la retroalimentación profesorado-alumnado (Gómez Ruiz, Rodríguez Gómez, e Ibarra Sáiz, 2013). Para Marcelo y Vaillant (2009) la formación de alto impacto en el desarrollo profesional docente es aquella que procura que reflexionen activamente acerca de sus prácticas y las comparen con indicadores profesionales adecuados.

El profesorado tiene que ser un profesional que busque soluciones y, por lo tanto, tiene que reflexionar sobre su propia práctica para emprender los cambios que le lleven a transformar positivamente la realidad (Cano e Imbernón, 2003). La necesidad de preguntarse y valorar la realización de esa actividad, hace que la evaluación se dirija hacia un proceso de reflexión sobre su propia práctica. Esta reflexión será más eficaz si se acompaña de herramientas que le guíen y le ayuden a profundizar en su desarrollo. Es aquí donde el portfolio cobra todo su valor como una herramienta para la mejora docente, basada en el análisis de las acciones de enseñanza, aprendizaje y evaluación de competencias genéricas del docente. La competencia reflexiva es un elemento necesario para la realización del portfolio, ya que le va confiriendo una cultura de reflexión sobre su práctica educativa, además de desarrollar habilidades metacognitivas para la autoevaluación.

El portfolio es una herramienta para el aprendizaje que integra la enseñanza y la evaluación y se emplea para una evaluación longitudinal de procesos y productos (Dochy, Segers y Dierick, 2002). Su finalidad principal es reunir trabajos que permitan el seguimiento y la evaluación del proceso de aprendizaje en el logro de resultados deseados (Rey Sánchez y Escalera Gámiz, 2011).

Según la revisión de la literatura, distintas investigaciones (Barragán, 2005; Cabero, López y Llorente, 2012; Mellado Hernández, 2010), consideran el portfolio como un instrumento 
de enseñanza, aprendizaje y evaluación caracterizado por recopilar de manera reflexiva las evidencias de aprendizaje de estudiantes y docentes. Sus características principales se basan en: mostrar la evolución del proceso de desarrollo profesional; estimular la experimentación, la reflexión y la investigación del alumnado; reflejar el punto de vista personal del estudiante sobre su aprendizaje; evidenciar los momentos claves del proceso de enseñanza y aprendizaje (problemas, soluciones o logros).

La unión de esta herramienta a las tecnologías de la información y la comunicación (TIC) se considera de especial relevancia para monitorear el avance en el proceso de enseñanzaaprendizaje. Según Barberá (2008:12), un e-portfolio es "un sistema digital que permite a los usuarios documentar competencias, eventos, planes o productos que son relevantes para ellos, así como dejar su evolución a lo largo del tiempo elegido”. Las herramientas digitales mejoran la interacción entre profesores y alumnos rompiendo la barrera de espacio y tiempo y permitiendo incorporar diferentes formatos audiovisuales y textuales. Además, la incorporación de las tecnologías educativas al aula, ha permitido tener más facilidad de acceso a la información y de inmediatez de los contenidos, estableciendo la competencia digital del profesorado como un aspecto necesario en su formación. En el estudio que nos ocupa, los estudiantes son los propios profesores y directores de centros educativos y, en consecuencia, la finalidad del e-portfolio está destinada al proceso de desarrollo profesional docente.

La experiencia práctica en la Universidad de Sevilla (Barragán, 2005) con la utilización del portfolio concluye la importancia del uso de infraestructuras tecnológicas y recursos informáticos que permitan incorporar de forma real las TIC como herramientas cotidianas de trabajo. Esta experiencia ofrece un mayor acercamiento al alumnado, permitiendo una mayor adaptación de la enseñanza a sus necesidades. Las evidencias recogidas en el portfolio demuestran que su uso promueve el desarrollo de la capacidad de reflexión y análisis crítico, y permite obtener un mayor control del trabajo realizado por el alumnado.

Martínez Olmo (2008) distingue dos tipos de portfolios según su funcionalidad: los portfolios sumativos, cuando el interés de su elaboración se centra en la certificación o selección, y los formativos cuando el interés recae principalmente en la autoevaluación y el proceso de aprendizaje. Estos últimos son en los que se fundamenta este estudio, ya que como señala Barrett (2009) el portfolio como proceso está dirigido a una actividad de aprendizaje.

Entre sus principales ventajas se encuentran la flexibilidad para poder ser aplicado a cualquier nivel educativo y la posibilidad de integrar elementos de autoconocimiento, autoevaluación y coevaluación (Elizalde Lora y Reyes Chavez, 2008). Entre las limitaciones en la utilización del portfolio, Barberá (2005) enumera: a) la falta de cultura reflexiva, nada o poco extendida en el sistema educativo que se centra, principalmente, en 
trabajar la cantidad de contenidos más que en su proceso; b) el desconocimiento de la herramienta, lo que hace que se generen inseguridades y, a menudo, la necesidad continua de feedback de su trabajo; c) puede producir un gasto excesivo de tiempo, debido a su alta personalización; d) la evaluación final hace que los criterios tengan que diseñarse de manera consensuada y, por último, e) los conocimientos informáticos juegan un papel decisivo a la hora de la realización de un portfolio digital.

Basándonos en las propiedades que Cano e Imbernón (2003) señalan del portfolio como herramienta para el desarrollo profesional docente, se destaca: la ayuda a la reflexión sobre lo que se hace, por qué se hace y cómo se hace. El portfolio facilita la observación de la evaluación del proceso de enseñanza-aprendizaje y a ser más consciente de ello. La herramienta permite compartir conocimiento, dado que tiene un carácter público. Y su uso también permite solicitar reconocimiento o premio,en torno a las evidencias seleccionadas durante el periodo de creación.

Este trabajo se centra en la utilización del e-portfolio en el desarrollo profesional docente no universitario, vinculado a un proceso de formación continuo que haga al profesor reflexionar sobre su práctica docente y desarrolle las competencias necesarias implicadas en su desarrollo.

\section{Objetivos}

El objetivo general del presente estudio se centra en analizar la percepción del profesorado en torno a la herramienta de e-portfolio para su desarrollo profesional.

De manera específica se propone valorar la competencia reflexiva como aspecto característico para la elaboración del e-portfolio, así como exponer el potencial de la herramienta e-portfolio teniendo en cuenta las ventajas y limitaciones experimentadas durante su elaboración.

\section{Desarrollo de la innovación}

El desarrollo de la innovación ha consistido en la elaboración y puesta en práctica del eportfolio como herramienta de aprendizaje y evaluación, entre un grupo de profesores y personal directivo de centros concertados de educación infantil, primaria, secundaria y bachillerato. Para ello, se lleva a cabo un programa innovador de desarrollo profesional llamado "Profesores en acción", en el que han participado 75 centros educativos diferentes, repartidos por todo el territorio nacional, abarcando 13 Comunidades Autónomas. Todos ellos comparten la necesidad de cambio y se encuentran en procesos de transformación educativa. Como parte de este programa, entre los meses de Enero y Abril de 2015, se

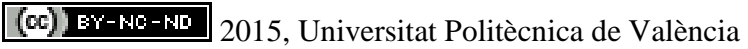

Congreso In-Red (2015) 
forma a 150 profesores y directivos en metodologías innovadoras para que puedan ser implementadas en sus centros El programa de formación, incluye cuatro módulos presenciales de formación dirigidos a mejorar la calidad docente. Los estudiantes (profesores y directores asociados al programa) realizan un curso de construcción y uso del e-portfolio de 20 horas de forma paralela. A medida que se desarrollan los módulos, los profesores y directores, convertidos ahora en alumnos, elaboran el $e$-portfolio en base a los contenidos expuestos en el curso.

Los participantes se encuentran divididos en seis grupos de 25 alumnos (tres grupos de profesores y tres grupos de directivos), cada uno con un profesor-tutor asociado, cuyo rol será de guía y feedback que tutorice todo el proceso de elaboración del e-portfolio.

Teniendo en cuenta las limitaciones enunciadas por Barberá (2005) se diseñan las propuestas de acción dirigidas a solventarlas durante la elaboración del portfolio (ver tabla 1).

Tabla 1. Propuestas de actuación diseñadas para el curso de e-portolio

\begin{tabular}{|c|c|}
\hline Limitaciones & Propuesta de actuación \\
\hline Falta de cultura reflexiva. & $\begin{array}{l}\text { Se ofrecen tiempos de reflexión sobre su } \\
\text { formación para facilitar la visión del proceso de } \\
\text { aprendizaje, a través de preguntas claras y } \\
\text { directas }\end{array}$ \\
\hline $\begin{array}{l}\text { Inseguridad en la elaboración del } \\
\text { portfolio. }\end{array}$ & $\begin{array}{l}\text { Se utilizan tutorías digitales a través de diferentes } \\
\text { instrumentos (foros, correo electrónico...) así } \\
\text { como la visualización de videotutoriales. }\end{array}$ \\
\hline $\begin{array}{l}\text { Gasto excesivo de tiempo en } \\
\text { personalizar la herramienta. }\end{array}$ & $\begin{array}{l}\text { Proceso de aprendizaje guiado a través de fechas } \\
\text { de entrega orientativas. }\end{array}$ \\
\hline $\begin{array}{l}\text { La evaluación final hace que los } \\
\text { criterios tengan que diseñarse de } \\
\text { manera consesuada y por último }\end{array}$ & $\begin{array}{l}\text { Se diseñan y presentan desde el inicio rúbricas de } \\
\text { evaluación para faciltar tanto la evaluación, como } \\
\text { de la autoevaluación. }\end{array}$ \\
\hline Baja competencia digital. & $\begin{array}{l}\text { Se mide la competencia digital antes de la } \\
\text { elaboración, y sugerir qué herramienta digital es } \\
\text { la más adecuada para la realización del e-portfolio }\end{array}$ \\
\hline
\end{tabular}

\subsection{Elaboración del e-portfolio del estudiante}

Son los propios profesores y directores, como alumnado del curso, los que elaboran el $e$ portfolio a través de las herramientas digitales gratuitas Google Sites o Mahara. Según su 
competencia digital, el estudiante selecciona la más adecuada para la construcción de su portfolio (Google Sites requiere un nivel de competencia digital medio-bajo y Mahara un nivel de competencia digital medio o alto). Esta selección se debe a las características técnicas de cada una de estas herramientas digitales, Google Sites utiliza una tecnología más intuitiva para el usuario, frente a la elaboración de contenidos digitales en Mahara.

El curso online cuenta con contenidos que les van guiando durante el proceso, junto con videotutoriales, además, de la disposición de un foro, atendido por su profesor-tutor, para consultar las dudas que puedan surgir durante su elaboración. En la programación semanal del curso se pautan las entregas de actividades en una fecha aproximada.

La estructura del portfolio digital se encuentra dividida en varias páginas y subpáginas. Al tratarse de una herramienta personal, esta estructura es una recomendación, y en ningún caso es obligatoria o cerrada. La recomendación que se realiza para la construcción del $e$ portfolio es:

- Portada: El alumno personaliza con una imagen o título.

- Perfil: Se describe personalmente, incluyendo sus intereses y objetivos.

- Currículum: Breve currículum de su trayectoria profesional.

- Mi PLE: Los Entornos Personales de Aprendizaje (Personal Learning employed, PLE) permiten al usuario guardar archivos y materiales de interés, así como sus aplicaciones personales. Estos entornos se proponen como alternativa de la herramienta portfolio (Barberá, Gewerc Barujel y Rodríguez Illera, 2009), debido al uso que se está dando de Internet. En este sentido, se integra formando una parte más del mismo y completándolo.

- Mi blog: También llamado "diario de aprendizaje”, consiste en resumir lo que se aprendió durante el módulo presencial. Para ayudar en esta tarea pueden valerse de las rutinas de pensamiento, "palabra-idea-frase", utilizadas para favorecer el pensamiento. En experiencias como la de la Universidad Pablo de Olavide (Martínez Gimeno y Hermosilla Rodríguez, 2010) utilizan el blog como herramienta para la realización de portfolios. En este caso, se inserta dentro del mismo como un diario de actualización periódica.

- Recopilación y selección de las evidencias de cada módulo. Se divide en recopilar la evidencias de aprendizaje del módulo presencial de la formación y en seleccionar una de esas evidencias por cada uno de los módulos presenciales trabajados durante el curso presencial "Profesores en Acción". Se les facilita una serie de preguntas para ayudarles a reflexionar.

- Evaluación: Realización de una autoevaluación y coevaluación de otros portfolios mediante una rúbrica de evaluación. Este instrumento intenta ser una ayuda para definir lo que espera el profesor que aprendan, y disponer de criterios concretos sobre la valoración de su trabajo (De la Serna, Rivas y Domínguez, 2007). 
El seguimiento y evaluación del e-portfolio se hace de manera continua a través de la retroalimentación del profesor-tutor, bien resolviendo sus dudas en los foros correspondientes, bien a través de la puntuación y comentarios que obtienen de la corrección de cada actividad entregada.

\subsection{Evaluación de la innovación}

La recogida de información sobre la situación de partida de los docentes y directores se realiza a través de una evaluación previa al curso online de e-portfolio, centrado en el análisis específico de la identificación del nivel de competencia digital, necesario para utilizar la herramienta online con la que se elabora el e-portfolio y el uso previo de la herramienta, así como la percepción que los profesores y directores tienen sobre el uso del portfolio antes del proceso formativo.

Para el análisis de la competencia digital de los estudiantes (profesores y directores) al curso de e-portfolio se utiliza un cuestionario online construido ad hoc. Para su elaboración, se toma como referencia el marco común de competencia digital docente (INTEF, 2013). El cuestionario recoge información sobre el uso del portfolio y se evalúa el nivel de competencia digital. En cuanto a la competencia digital se miden aquellas dimensiones estrechamente relacionadas con áreas de competencia de información, comunicación y creación de contenidos. Estas son: en cuanto a navegación, búsquedas y filtros de información en Internet; almacenamiento y recuperación de la información; compartir información y contenidos; y desarrollo de contenidos digitales.

Para la recogida de datos sobre la utilidad del portfolio, se elabora un cuestionario ad hoc con un coeficiente de fiabilidad de 0,916 , obtenido a través del método de consistencia interna basado en el Alfa de Cronbach. Este cuestionario es sometido a una evaluación interjueces. Los jueces, vinculados a distintas universidades de educación, evaluaron la validez, claridad y relevancia de cada ítem en una escala de 1 a 6 ; donde uno indica un ítem nada relevante y seis totalmente relevante. Además, se tuvieron en cuenta las observaciones y valoraciones añadidas por los jueces.

\section{Resultados}

La mayoría de los participantes, docentes y directores de enseñanza no universitaria, tienen una experiencia docente (Figura 1) superior a 15 años (48\%), entre 10-14 años se encuentra el 22\%, entre 5-9 años el 25\% y con una experiencia docente menor a 5 años el 5\%. 
Martínez-De la Muela, A., García-García, M., Arteaga-Martínez, B.

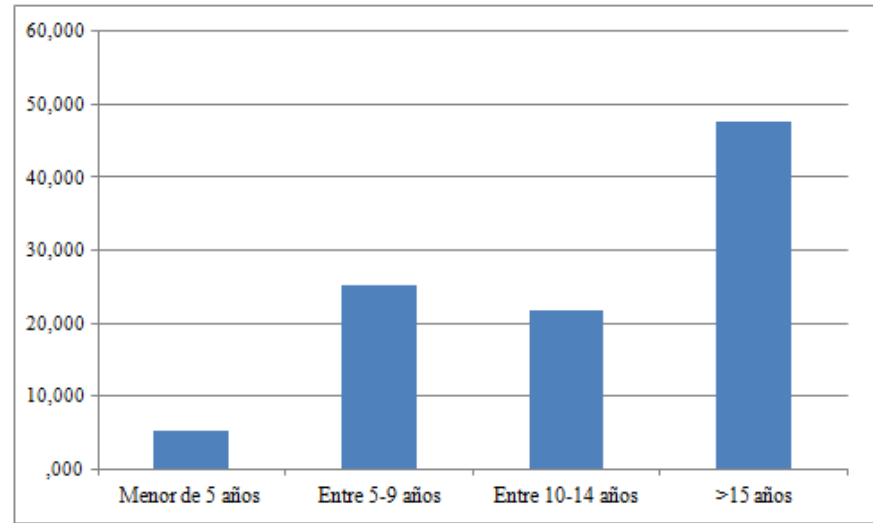

Fig. 1 Porcentaje de experiencia docente

El 11\% de profesores y directores han realizado un portfolio con anterioridad, ya sea en papel o en soporte digital mientras que el $89 \%$ de los participantes nunca lo han realizado. Sólo 2 personas lo tienen en activo actualmente. Los estudiantes se sitúan en competencia digital media-baja, siendo el desarrollo de contenidos digitales, con una media de 1,430 ( $\mathrm{n}=147 ; \sigma=, 688)$ y el modo de compartir materiales digitales a través de la red con media 1,563 ( $\mathrm{n}=147 ; \sigma=, 753$ ) las características de la competencia digital más bajas (nivel bajo).

Los resultados muestran la percepción que poseen los estudiantes (profesores y directores de centro) del e-portfolio para su desarrollo profesional docente, en cuanto a tres aspectos principales: 1) su capacidad para facilitar el trabajo cooperativo con los compañeros; 2) su capacidad de reflexión; y 3) su capacidad de razonamiento crítico. Así mismo, se analiza con mayor profundidad la competencia reflexiva, como la principal característica de la herramienta, y se valoran las aportaciones de los estudiantes en cuanto a las ventajas e inconvenientes que estén pudiendo surgir durante su elaboración. De los análisis resultados destacaremos los siguientes:

El 13\% responde que facilita totalmente el trabajo cooperativo entre compañeros, el $43 \%$ que lo facilita mucho y el $26 \%$, bastante. Solo el $7 \%$ cree que lo facilita algo y el $11 \%$ no sabe.

El 48\% responde que el uso del portfolio facilita totalmente la capacidad reflexiva para su desarrollo profesional, el $31 \%$ que lo facilita mucho y el $13 \%$ que lo facilita bastante. Solo el $3 \%$ considera que facilita algo y el $5 \%$ no sabe.

Por último, en cuanto al desarrollo de la capacidad de autocrítica, el $43 \%$ responde que facilita mucho la capacidad de autocrítica, el $30 \%$ que lo facilita totalmente y el 19\% que lo facilita bastante. Solo el $1 \%$ piensa que facilita algo y el $7 \%$ no sabe.

\section{(cc)) BY-NC-ND 2015, Universitat Politècnica de València}

Congreso In-Red (2015) 
En la figura 2 se distingue la capacidad reflexiva como la competencia más valorada del $e$ portfolio para el desarrollo profesional docente, con una valoración media de 5,391 (n=92; $\sigma=, 901)$. La competencia de trabajo cooperativo muestra los datos más dispersos respecto a la media ( $\mathrm{n}=92 ; \mu=4,957 ; \sigma=1,047)$.

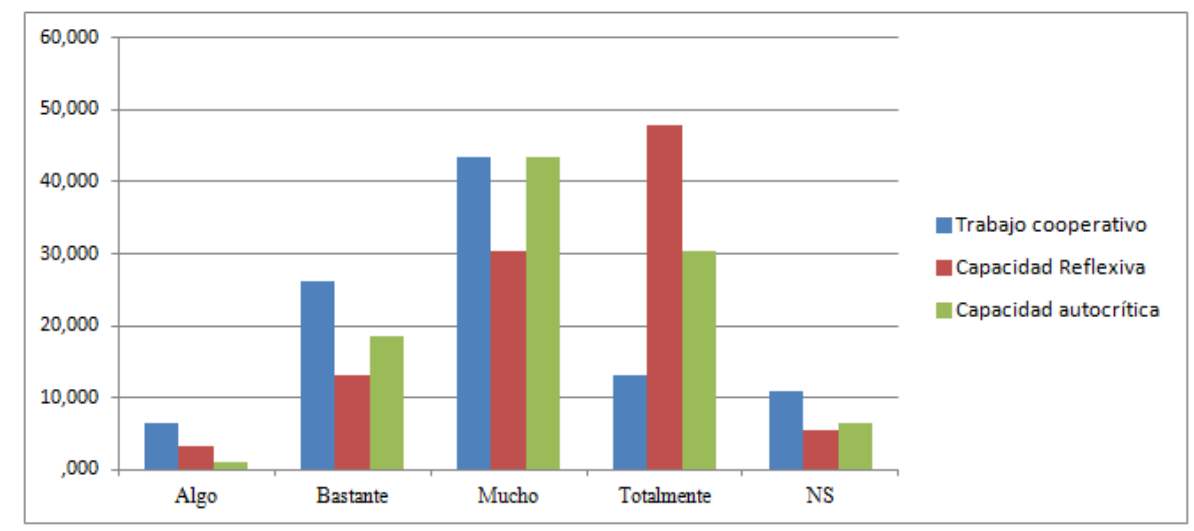

Fig. 2. Porcentaje de percepción de los profesores y directores en competencia digital

Dado que la capacidad reflexiva se muestra como la competencia percibida por los docentes como más importante entre las señaladas como de utilidad del portfolio, realizamos un análisis particular de ella de forma gráfica que expresa las valoraciones en cada uno de los ítems del cuestionario que la evalúan (Figura 3).

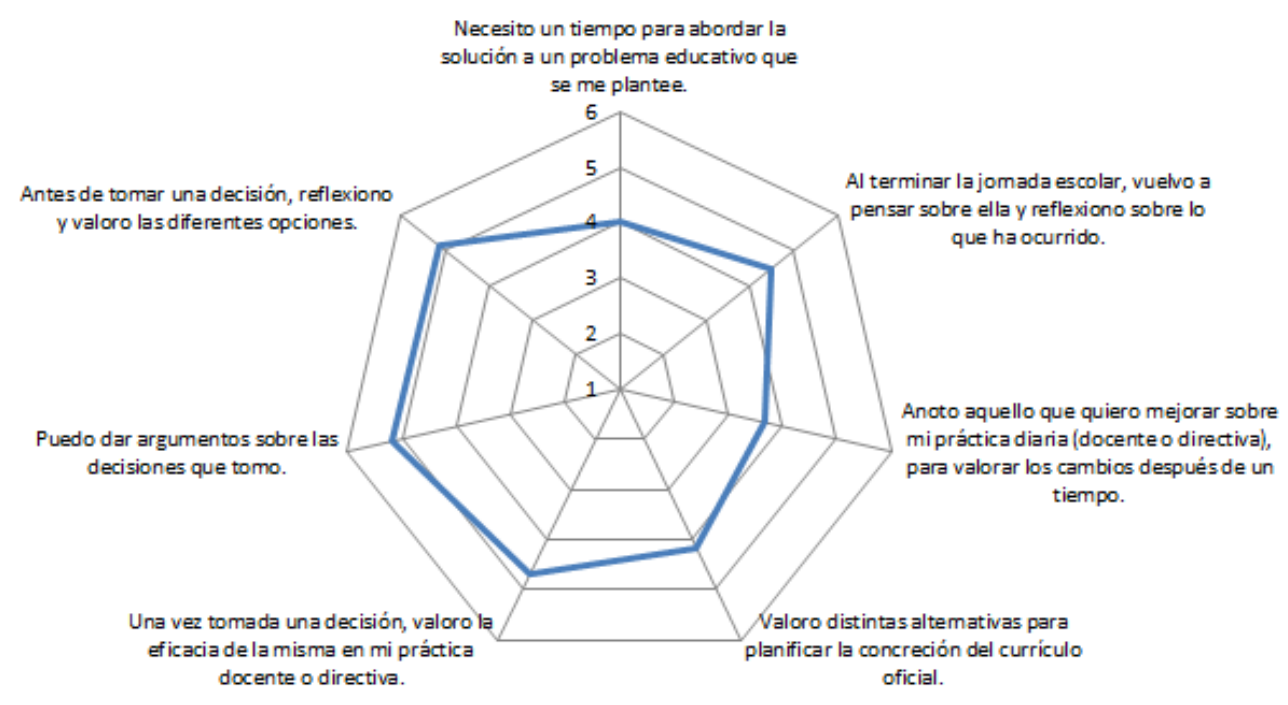

Fig. 3. Percepción de la competencia reflexiva 
Destacando como el ítem de mayor variabilidad en la respuesta "anoto aquello que quiero mejorar sobre mi práctica diaria (docente o directiva), para valorar los cambios después de un tiempo" (n=92; $\mu=3,663 ; \sigma=1,1414)$.

Cabría pensar que esta percepción de la capacidad reflexiva, estuviese correlacionada (RhoSpearman) con los años de experiencia laboral, sin embargo entre los ítems del cuestionario que recogen esta capacidad únicamente dos de ellos muestran una relación significativa (p$\mathrm{v}>, 05$ ), "necesito un tiempo para abordar la solución a un problema educativo que se me plantee" ( $\mathrm{r}=, 205 ; \mathrm{n}=92)$ y "valoro distintas alternativas para planificar la concreción del currículo oficial” ( $\mathrm{r}=, 206 ; \mathrm{n}=92)$.

De las reflexiones aportadas por los participantes (profesores y directores de centros) durante la elaboración del e-portfolio, se recogen comentarios sobre qué les ha supuesto la elaboración de la herramienta parece que perciben su utilidad, tanto para su formación como para usarlo posteriormente en el aula. MLM al respecto comenta: "la verdad es que le estoy viendo muchas posibilidades a esta herramienta que, hasta ahora, no me había metido de lleno en ella. Tengo ganas de seguir avanzando porque creo que, en cuanto tenga el contenido entero, voy a aplicarla en el aula”. En cuanto al desarrollo de las competencias que la teoría relaciona con el desarrollo del portfolio, la capacidad reflexiva se apoya en comentarios como el de C.L.P: "La actividad me está ayudando a aprender, conectar y profundizar, para realizar un e-portfolio, nunca lo había hecho, siempre hacia una recogida de actividades sin más ampliación, ni actividades que evidenciaran lo trabajado". O las aportaciones de E.G: "Ha sido una interesante reflexión al mismo tiempo que mejora la autoestima porque he sido más consciente de mi constante necesidad y búsqueda de cambios adaptándome a la realidad del mundo en que vivimos”. El e-portfolio les ayuda a desarrollar habilidades metacognitivas necesarias para su práctica docente, como expresa el estudiante J.J.B.B: "Me parece un método de autocorrección y autoevaluación muy válido para los proyectos y actividades innovadoras que estamos llevando a cabo en el centro".

En cuanto a las limitaciones, se señalan algunas de las percibidas por las reflexiones, como la del tiempo y espacio comentada por E.M: "Dadas mis circunstancias personales y profesionales no he contado con el tiempo y espacio adecuado para llevar a cabo tal actividad. Me quedo con que he iniciado un camino de transformación que aunque tal vez sólo esté iniciado, junto con mi equipo he de seguir”. La falta de conocimiento o, quizás, la inseguridad, señaladas por M.A.B.C, quien comenta "Al revisar el trabajo, me he dado cuenta de que faltaban algunas actividades. Creo que ya las he completado todas. Quizá todavía me lío un poco con el tema de las evidencias, probablemente les daré otra vuelta”. 
El e-portfolio como herramienta para el desarrollo profesional docente

\section{Conclusiones}

El portfolio es un recurso que posibilita que la persona muestre y transforme en evidencias aquello que ha aprendido (Barberá, Gewerc Barujel y Rodríguez Illera, 2009) pudiendo favorecer el desarrollo de competencias genéricas necesarias para su desarrollo profesional docente. Los profesores y directores perciben el e-portfolio como una herramienta útil para desarrollar el trabajo en equipo, el razonamiento crítico y la competencia reflexiva. Respecto a esta última, la reflexión es la característica del e-portfolio que más valoran para su desarrollo profesional docente. Esta competencia les ayuda a conectar información, valorar su práctica y evidenciar su aprendizaje, favoreciendo el desarrollo de habilidades metacognitivas como la autoevaluación. Contrastando con la tarea diaria de recogida y anotación de reflexiones que realizan durante su práctica en el centro, este aspecto es el más disperso, pudiendo pensar que necesitan de unas herramientas que les faciliten esta recopilación y selección de evidencias. En este sentido, señalar que casi la totalidad de los participantes, no habían construido un portfolio con anterioridad, lo que significa establecer un mayor énfasis en los contenidos teóricos de esta herramienta o en la ampliación del tiempo de formación.

En un momento de transformación innovadora donde las tecnologías tienen presencia en muchas ocasiones de la práctica educativa, la competencia digital supone una atención especial para la elaboración del e-portfolio. Los profesores y directores presentan una competencia digital media-baja, lo que dificulta el manejo de herramientas digitales para la elaboración del e-portfolio, atendiendo a las dimensiones con una valoración más baja su capacidad para compartir y generar contenidos web. Para los estudiantes, la falta de tiempo se señala como una de las limitaciones más determinantes para el desarrollo del eportofolio. No obstante, los profesores y directores señalan el e-portfolio como una herramienta de futura aplicación en el aula.

La formación en competencias a través de herramientas, como el e-portfolio, que ayuden a evaluar la práctica y guiar la mejora del desarrollo profesional, se convierten hoy en día en el punto de mira para una calidad docente y directiva, adecuada a las escuelas del siglo XXI.

\section{Agradecimientos}

Este trabajo se ha realizado gracias al Departamento de Innovación Pedagógica de Escuelas Católicas, organizador del programa "Profesores en acción" y al equipo de investigación de UNIR "Educación Personalizada en la Era Digital” (EPEDIG). Esta actividad ha sido parcialmente financiada por UNIR Research (http://research.unir.net), Universidad Internacional de la Rioja (UNIR, http://www.unir.net), dentro del Plan Propio de Investigación, Desarrollo e Innovación [2013- 2015]. 
Martínez-De la Muela, A., García-García, M., Arteaga-Martínez, B.

\section{Referencias}

BARBERÁ, E. (2005). La evaluación de competencias complejas. La práctica del portafolio. EDUCERE. Revista Venezolana de Educación,.(31). pp.497-503.

< http://www.saber.ula.ve/browse-title> /> [Consulta:20 de Mayo de 2015]

BARBERÁ, E. (2008). El estilo e-portafolio. Barcelona: UOC.

BARBERÁ, E., GEWERC BARUJEL, A. y RODRÍGUEZ ILLERA, J.L. (2009). Portafolios electrónicos y educación superior en España: Situación y tendencias. RED, Revista de Educación a Distancia. Número monográfico VIII, pp.1-13.

$<$ http://www.um.es/ead/red/M8/> [Consulta:20 de Mayo de 2015]

BARRAGÁN, R. (2005). El portafolio metodología de evaluación y aprendizaje de cara al nuevo espacio de educación superior. Una experiencia práctica en la universidad de Sevilla. Revista Latinoamericana de Tecnología educativa, 4(1), pp. 121-139.

BARRETT, H. (2009). Balancing the Two Faces of E-Portfolios.

<http://electronicportfolios.org/balance/Balancing2.htm> [Consulta:14 de Mayo de 2015]

BISQUERRA, R. (2003). Educación emocional y competencias básicas para la vida. Revista de Educación Educativa (RIE), 21 (1), pp. 7-43.

$<$ http://revistas.um.es/rie/article/view/99071> [Consulta:12 de Mayo de 2015]

CABERO, J., LÓPEZ, E. y LLORENTE, M. (2012). E-Portafolio universitario como instrumento didáctico 2.0 para la reflexión, evaluación e investigación de la práctica educativa en el espacio europeo de educación superior. VEsC. Revista Virtualidad, Educación y Ciencia, 3(4), pp.27-45.

$<$ http://revistas.unc.edu.ar/index.php/vesc/article/view/1886> [Consulta:26 de Diciembre de 2014]

CANO, E. e IMBERNÓN, F. (2003). La carpeta docente como instrumento de desarrollo profesional del profesorado universitario. Revista universitaria de formación del profesorado, 17 (2), pp.43-51 $<$ http://www.aufop.com/aufop/uploaded_files/articulos/1241444741.pdf> [Consulta:14 de Mayo de 2015]

DAY, C. (1999). Developing Teachers. The Challenges of Lifelong Learning. London: Falmer Press.

DE LA SERNA, M. C., RIVAS, M. R., y DOMÍNGUEZ, J. A. A. (2007). E-portafolio en el practicum: un modelo de rúbrica. Comunicación y pedagogía: Nuevas tecnologías y recursos didácticos, 218, pp.8-14.

$<$ http://bit.ly/1cgC19M> [Consulta:20 de Mayo de 2015]

DOCHY, F., SEGERS, M. y DIERICK, S. (2002). Nuevas vías de aprendizaje y enseñanza y sus consecuencias: una nueva era de evaluación. Revista de Docencia universitaria, 2(2), pp.13-29 $<$ http://revistas.um.es/redu/article/view/20051/19411> [Consulta:19 de Mayo de 2015]

ELIZALDE LORA, L. y REYES CHAVEZ, R. (2008). Elementos clave para la evaluación del desempeño de los docentes. REDIE, 10. pp.1-13.

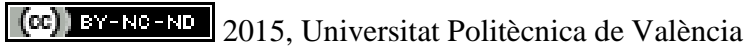

Congreso In-Red (2015) 
El e-portfolio como herramienta para el desarrollo profesional docente

$<$ http://www.scielo.org.mx/scielo.php?script=sci_arttext\&pid=S160740412008000300004\&lng=es\&nrm=iso> [Consulta:19 de Mayo de 2015]

GARCÍA, M. E. C. (2008). La evaluación por competencias en la educación superior. Profesorado. Revista de curriculum y formación de profesorado, 12 (3), pp. 1-16.

$<$ http://www.ugr.es/ recfpro/rev123COL1.pdf> [Consulta:25 de Mayo de 2015]

GÓMEZ-RUIZ, M. Á., RODRÍGUEZ-GÓMEZ, G. y IBARRA-SÁIZ, M. (2013). Desarrollo de las competencias básicas de los estudiantes de Educación Superior mediante la e-Evaluación orientada al aprendizaje. RELIEVE-Revista Electrónica de Investigación y Evaluación Educativa, 19 (1), pp.1-17. <http://www.uv.es/relieve/v19n1/RELIEVEv19n1_1.htm> [Consulta:25 de Mayo de 2015].

GONZÁLEZ MAURA, V. (2006). El diario como instrumento de diagnóstico y estimulación del desarrollo profesional del profesorado. Revista Iberoamericana de Educación, 38, pp.1-14. $<$ http://www.rieoei.org/deloslectores/1248Gonzalez.pdf> [Consulta:25 de Mayo de 2015]

IMBERNÓN, F. (1994). La formación y el desarrollo profesional del profesorado. Hacia una nueva cultura profesional. Barcelona: Grao

INTEF (2013). Marco común de competencia digital docente. Madrid: Ministerio de Educación, Cultura y Deporte.

$<$.http://bit.ly/1dX2jix> [Consulta:15 de Mayo de 2015]

MARCELO, C. (2009). La evaluación del desarrollo profesional docente: de la cantidad a la calidad. Revista Brasileira de Formação de Professores, 1(1), pp. 43-70.

MARCELO, C y VAILLANT. D. (2009) Desarrollo profesional docente. ¿Cómo se aprende a enseñar?.Madrid: Narcea Ediciones.

MARCELO, C. (2011). "La evaluación del desarrollo profesional docente” en Veláz de Medrano, C., Vaillant, D. Aprendizaje y desarrollo profesional docente. Madrid: OIE. Fundación Santillana.

MARTÍNEZ OLMO, S. (2008) “El dossier de aprendizaje: Técnica de evaluación alternativa” en Cuadernos de docencia universitaria. Barcelona: Ediciones OCTAEDRO.

MARTÍNEZ GIMENO, A y HERMOSILLA RODRÍGUEZ, J.M. (2011). El blog como herramienta didáctica en el Espacio Europeo de Educación Superior. Pixel-Bit. Revista de Medios y Educación, 38, pp. 165-175.

< https://idus.us.es/xmlui/handle/11441/22638> [Consulta: 25 de Mayo de 2015]

MELLADO HERNÁNDEZ, M. E. (2010). Portafolio en línea en la formación inicial docente. Revista Electrónica De Investigación Educativa, 12(1), pp. 1-32.

<http://www.scielo.org.mx/scielo.php?pid=S1607-40412010000100007\&script=sci_arttext>

[Consulta: 18 de Mayo de 2015]

PEÑA, J.V (2003). Desarrollo profesional del docente universitario. Monografías Virtuales. Ciudadanía, democracia y valores en sociedades plurales. Línea temática: Universidad, profesorado y ciudadanía, 3. Madrid: OEI,

$<$ http://www.campus-oei/valores/monografias/monografia03/reflexiones03.htm> [Consulta:25 de Mayo de 2015]. 
Martínez-De la Muela, A., García-García, M., Arteaga-Martínez, B.

PROYECTO TUNNING (2003). Tuning Educational Structures in Europe. Informe Final. Fase I. Bilbao: Universidad de Deusto

REY SÁNCHEZ, E. y ÁGUEDA, M. E. G. (2011). "El portafolio digital un nuevo instrumento de evaluación." DIM: Didáctica, Innovación y Multimedia, 21, pp. 1-10.

<http://www.raco.cat/index.php/DIM/article/view/247586> [Consulta: 19 de Mayo de 2015] 